\title{
TCP Adaptation for Vertical Handoff Using Network Monitoring
}

\author{
Faraz Idris Khan and Eui Nam Huh \\ Internet Computing and Security Lab \\ Department of Computer Engineering, Kyung Hee University, 449-701 Yongin, \\ South Korea \\ \{faraz, johnhuh\}@khu.ac.kr
}

\begin{abstract}
Next Generation Network envisions convergence of different wireless networks to provide ubiquitous communication environment to the mobile user. The ubiquity is achieved by enabling a mobile user to switch to a network with better QoS (Quality of Service), by a mechanism called vertical handoff. We propose a vertical handoff management architecture which handles TCP adaptation and ensure efficient utilization of mobile terminal resource i.e. memory. The adaptation is achieved by configuring TCP socket buffer according to the available network resource i.e. bandwidth. The simulation results show that TCP utilizes the bandwidth and memory efficiently by employing our TCP adaptation strategy.
\end{abstract}

Keywords: TCP adaptation, adaptive network QoS, vertical handoff, congestion control, TCP performance.

\section{Introduction}

Mobile communications and wireless networks are developing at a fast pace. The increasing number of mobile subscribers and terminals are the real life evidence of the fast growing development in the area of wireless communication. As a resultant effect an increasing demand of IP based services (e.g. e-commerce) and IP related enormous applications (e.g. WWW and email), the demand for wide band data access through wireless medium is created. Integration of different heterogeneous networks is able to diminish the wideband data access problem and also capable of minimizing the deployment cost for completely new infrastructure and access.

Among different wireless access technologies, WLAN [3] and Cellular system [4] are getting momentum during last couple of years. WLAN is mostly used to serve the hotspot region like airports, cyber cafes, coffee shops, hospitals, schools etc. for its huge data rate (11 Mbps for 802.11b, 54 Mbps for 802.11a and 802.11g) and relatively low cost.

In telecommunication domain cellular system remains the most popular wireless access technologies in communication domain. The development of cellular system resulted into different generations like 1G, 2G (GSM, DAMPS), 2.5G (GPRS, EDGE) followed by $3 \mathrm{G}$ (UMTS). In 1992, International Telecommunication Union (ITU) has issued International Mobile Telecommunication for year 2000 (IMT-2000) which defines the basic characteristics of $3 \mathrm{G}$. The advantages of cellular system are wide 
coverage, well known voice service whereas low data rate and cost relative to WLAN are few of the limitations.

In order to provide the communication platform for pervasive systems the capabilities of both WLAN and cellular systems will be combined. This can be achieved with the overlay architecture in which different networks overlap each other. The objective is to provide good Quality of Service (QoS) to the mobile user all the time. This is achieved by a mechanism called vertical handoff which enables a mobile user to switch to a different network. The application experiences degradation in QoS due to changing network parameters such as RTT and packet loss rate. A handoff decision is made to switch to a better network which provides better QoS to the mobile user. The decision of switching to another network considers user profile, device profile and application profile in order to provide seamless service to the end user [2]. There are two kinds of vertical handoff situation that might occur. Either the mobile terminal switches from a high data rate network to low data rate network or it moves from low data rate network to high data rate network. In the former case TCP congestion window does not open up fully due to which the bottleneck remains underutilized. While in the latter case the memory allocated to a TCP connection is wasted as the new network is a low data rate network. In order to provide undisrupted service to the end user there are various vertical handoff management architecture proposed in literature [1][2][4][9] which mostly considers content adaptation after vertical handoff. There are few architecture proposed such as in [9] which incorporates TCP adaptation but they require modification in TCP.

Thus in this paper we propose vertical handoff management architecture, providing undisrupted service to the end user by incorporating TCP adaptation module.The TCP adaptation module requires no modification in TCP code but requires application level configuration of socket buffer size.

The structure of the paper is as follows. Section 2 describes the related work on vertical handoff architecture and provides a brief overview of current research considering TCP performance in wireless network and TCP buffer tuning techniques. Section 3 describes the proposed vertical handoff architecture. Section 4 contains the simulation results of the TCP adaptation logic considering vertical handoff scenarios.

\section{Related Work}

This section is divided into three subsections. In subsection1 vertical handoff management architecture is discussed. Subsection 2 contains work related to wireless TCP considering mobility and its adaptation in handoff scenario.

\subsection{Vertical Handoff Management Architecture}

Helal et al [1] proposed an architecture which considers application level adaptation and handles vertical handoff mobility by employing Mobile IP. Applications participate fully in vertical handoff decision process by making its need known which helps in application level adaptation.

Balasubramaniam et. al [2] presented a vertical handover as an adaptation method supporting pervasive computing. Adaptation is carried on multimedia streams upon a 
change in context which is defined by dynamic network and application profile. Vertical handoff decision is carried out by weighing the decision parameters employing AHP (Analytical Hierarchy Process) [3] and then calculating the quality of each network in order to decide the best network.

Di Caor et. al [4] proposed a strategy of optimized seamless handover applied in WiOptimo system in which the network parameters used for vertical handoff decision are adapted according to the context. A cross layer mechanism is employed to detect the status of the connection. The vertical handoff can occur in the following cases

- Switching to another network occurs due to user's preference

- If a mobile terminal is moving to the edge of the network the signal strength might degrade and a handoff might be required

- The third case that causes switching in case the current network is not good enough to provide QoS to the running application

If we summarize the above work we can see that in all of the architectures a separate module to handle TCP adaptation is not taken into consideration. We propose a separate module in vertical handoff management architecture which handles TCP adaptation.

\subsection{Advances in TCP Adaptation and Mobility}

TCP is the most popular transport protocol employed in internet to control the smooth transmission of data by employing a buffering mechanism. It is designed with reliable network in mind. Thus TCP performs poorly in case of wireless network which often experiences packet losses due to mobility of the terminal which invokes congestion control algorithm to lower the transmission rate of the application. Thus in literature we can find enhancements in TCP such as in [5] [6] [7] which are proposed to differentiate between packet losses due to congestion and unreliable nature of the wireless link.

In [8] an FRA algorithm is proposed which probes the new link exponentially rather than staying in congestion avoidance phase with linear probing in case the capacity increase is large. In the opposite case they use explicit handoff notification (EHN) to tackle the packet loss due to sudden reduction in capacity. In [9] a mechanism for dynamically adjusting the congestion window is proposed to adapt TCP. The scheme shrinks the window less rigorously upon detection of packet loss during vertical handoff period in order to improve TCP throughput and retain the retransmission timeout value.

\subsection{Advances in TCP Buffer Tuning}

There are various mechanisms proposed in literature to improve TCP throughput by dynamically adjusting the socket buffer size.

In [11] a daemon called Work Around Daemon handles the tuning of the sockets by using ping for measuring the RTT of the path and pipechar to estimate the capacity $\mathrm{C}$ of the path. The daemon cannot cater the changing BDP due to mobility of the mobile terminal.

The first proposal for automatic TCP buffer tuning was [12]. The main objective of their work is to enable fair sharing of the kernel memory among multiple TCP 
connections. The sockets are configured by considering the present congestion window to calculate the BDP of the path. The mechanism will work well for a path with constant BDP rather than for varying BDP in case of vertical handoff. In some other works such as in [13] dynamic adjustment of socket buffer tuning is proposed. The shortcomings of these mechanisms are that they do not cater the changing RTT and bandwidth during vertical handoff situation.

\section{System Architecture}

The architecture is designed in a modular fashion with a middleware based approach. The device, application, user, current network and application QoS profile are considered to be stored in a context repository (CR).CR loads the QoS profile which is used by different networks for QoS provisioning. The decision engine (DE) monitors the context cache for triggering the handoff upon detection of context changes which can be degradation in QoS due to changes in network characteristics, disconnection from the current network etc. The feedback from DE triggers the TCP level adaptation which is handled by TCP adaptation module (TAM). Network Resource Monitoring Agent (NRMA) agent monitors the network resources such as available bandwidth, RTT which is used by TCP adaptation module. The TCP adaptation module is discussed in detail in subsection 3.1.

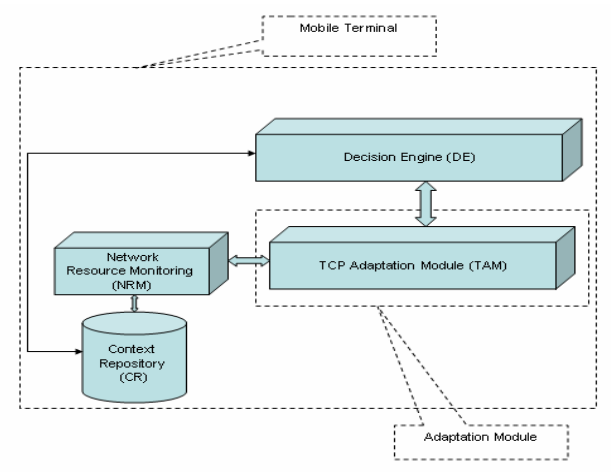

Fig. 1. System Architecture designed using a middleware based approach

\subsection{TCP Adaptation Module (TAM)}

The working of TAM is as follows. Let us denote the capacity of the network by $\mathrm{C}$ bps and the RTT by T sec. Thus in order to fully utilize the bandwidth of the network the socket buffer should be adjusted to at least $C \times T$. This will ensure that the TCP congestion window $W_{c}$ will open up to saturate the network. The size of the socket buffer can be represented by $\mathrm{S}$. The handoff situation can be of two types one is when a mobile terminal moves from high data rate network to low data rate network. The other is when a terminal moves from low data rate network to high data rate network. In either of the case the socket buffer size of the connection need to be adjusted 
according to the available bandwidth. Mathematically the socket buffer size of the application can be calculated as follows. If we represent the current network by $N_{c}$ and the new network by $N_{n}$ then the algorithm of TCP adaptation is shown in figure 2 .

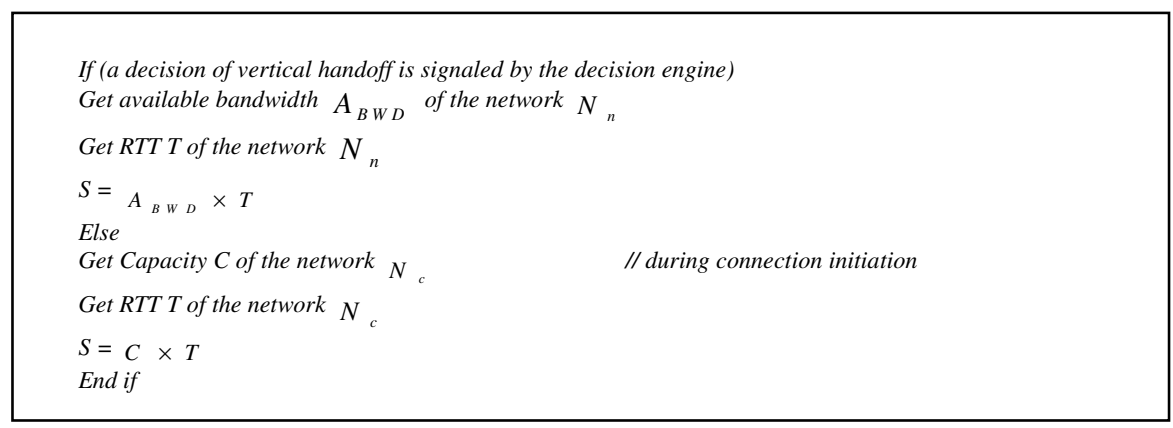

Fig. 2. TCP Adaptation Algorithm

\section{Simulation}

For TCP adaptation we simulated our proposed idea in $n s-2$ by implementing vertical handoff scenario. In this simulation we assume that the data rate with in $3 \mathrm{G}$ cellular network is $144 \mathrm{kbps}$ with end-to-end RTT $300 \mathrm{msec}$ and data rate with WLAN is 2Mbps with end-to-end RTT 100 msec.

In ns-2 simulation, the total duration was of 140 seconds. The mobile terminal moves from low data rate network to high data rate network. We assume that the vertical handoff occurs at $50 \mathrm{sec}$ and completes at $54 \mathrm{sec}$.

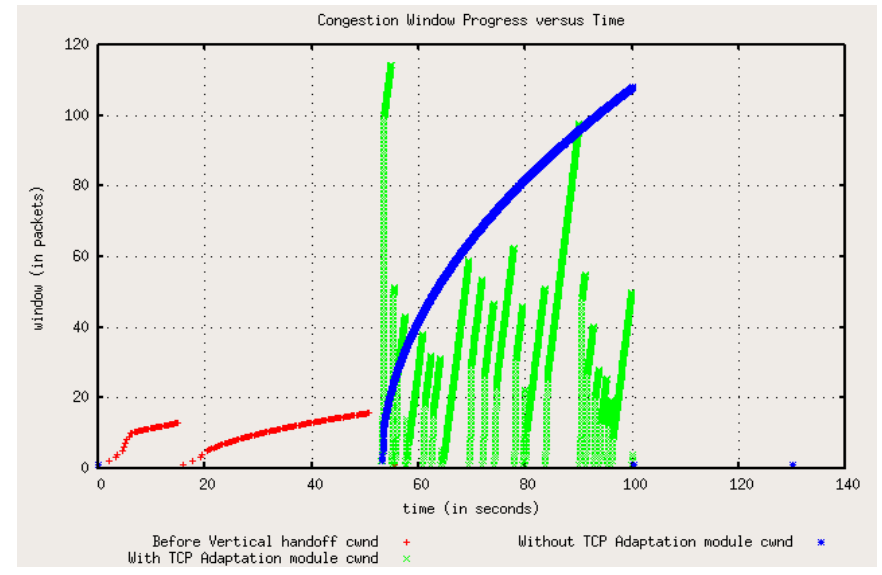

Fig. 3. Congestion Window Progress during vertical handoff from low data rate network to high data rate network 
There is a $65 \%$ improvement in the bottleneck utilization of the link. Figure 3 shows TCP window progress with and without the TCP adaptation module during vertical handoff. By configuring the buffer size, the newly available bandwidth is adapted swiftly but the TCP connection and it takes 4 seconds to hit maximum bandwidth as shown in figure 3 .

\subsection{High Data Rate Network to Low Data Rate Network}

Figure 4 and Figure 5 shows the memory utilization of a TCP connection with and with out TCP adaptation module. The memory remains largely underutilized because the socket buffer is not adjusted according to the bandwidth delay product. By applying TCP adaptation module the memory utilization of a mobile terminal is improved in our case by $40 \%$.

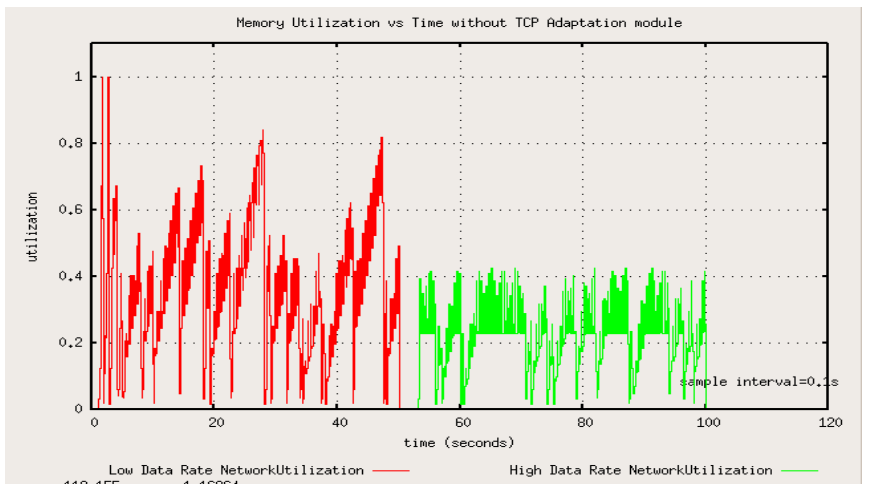

Fig. 4. Figure shows the memory utilization of the system with out TCP Adaptation module during handoff

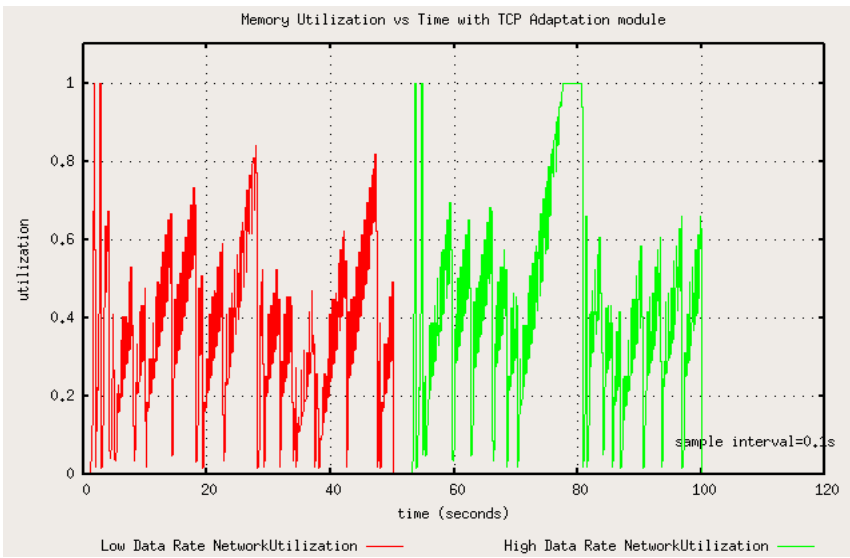

Fig. 5. Figure shows the memory utilization of the system with out TCP Adaptation module during handoff 


\section{Conclusion}

In this paper we propose vertical handoff management architecture with TCP adaptation for pervasive systems. We have incorporated a TCP adaptation module which uses socket buffer tuning mechanism to improve the TCP performance and maintain system performance by conserving system resources i.e. memory. The adaptation mechanism is initiated by feedback from DE in cooperation with NRMA which monitors the network resource. For our future work we will investigate the CPU resource consumption after vertical handoff situation. The ultimate objective is to develop a vertical handoff management architecture which provides QoS to the end user by maintaining application performance and ensure efficient utilization of resources.

Acknowledgements. This research was supported by MIC (Ministry of Information and Communication), Korea, under ITRC (Information Technology Research Center) support program supervised by the IITA (Institute of Information Technology Advancement). (IITA-2006-C1090-0603-0040).

\section{References}

1. Helal, S., Lee, C., Zhang, Y.G., Richard III, G.G.: An Architecture for Wireless LAN/WAN Integration. WCNC 2000 IEEE Wireless Communication and Networking Conference Chicago, 9 (2000)

2. Balasubramaniam, S., Indulska, J.: Vertical handover supporting pervasive computing in future wireless networks. Vol. 27 Computer Communications, 5 (2004) 708 - 719

3. Song, Q., Jamalipour, A.: A Network Selection Mechanism for Next Generation Networks. IEEE ICC'05, Seoul 5 (2005)

4. Gianni, A., Caro, D., Giordano, S., Kulig, M., Lenzarini, D.: A cross layer and autonomic approach to optimized seamless handover. IFIPS WONS France, 1 (2006)

5. Bakre, A., Badrinath, B.: I-TCP: Indirect TCP for mobile hosts. IEEE ICDCS (1995) 136-143

6. Brown, K. Singh, S.: M-TCP: TCP for mobile cellular networks. ACM Computer Comm. Review (CCR) Vol. 27(1997)

7. Balakrishnan, H., Padmanabhan, V.N., Seshan, S., Katz, R.H.: A comparison of mechanisms for improving TCP performance over wireless links. IEEE/ACM Trans. Networking Vol. 5. IEEE Computer Society (1997) 756-769

8. Chen, L.J., Yang, G., Sun, T., Sanadidi, M.Y., Gerla, M.: Enhancing QoS Support for Vertical Handoffs Using Implicit/Explicit Handoff Notification. QSHINE IEEE Computer Society (2005) 37

9. Kang, R.J., Chang, H.P., Chang R.C.: A seamless vertical handoff scheme. WICON IEEE computer society (2005) 64 - 71

10. Dunigan, T., Mathis, M., Tierney, B.: A TCP Tuning Daemon. SuperComputing: HighPerformance Networking and Computing Vol. 25. IEEE computer society, Baltimore Maryland (2002) 1 - 16

11. Semke, J., Madhavi, J., Mathis, M.: Automatic TCP Buffer Tuning. ACM SIGCOMM Vol. 28(1998) 315 - 323 
12. Gardner, M.K., Feng, W.C., Fisk, M.: Dynamic Right-Sizing in FTP (drsFTP): Enhancing Grid Performance in User-Space. IEEE Symposium on High-Performance Distributed Computing (2002) 42 - 49

13. Prasad, R., Jain, M., Dovrolis, C.: Socket Buffer Auto-Sizing for High-Performance Data Transfers. Journal of Supercomputing Vol. 1. (2003) 361-376 